\title{
Suprathermal electron acceleration during reconnection onset in the magnetotail
}

\author{
A. Vaivads ${ }^{1}$, A. Retinò ${ }^{2,3}$, Yu. V. Khotyaintsev ${ }^{1}$, and M. André ${ }^{1}$ \\ ${ }^{1}$ Swedish Institute of Space Physics, Uppsala, P.O. Box 537, 75121 Uppsala, Sweden \\ ${ }^{2}$ Laboratoire de Physique des Plasmas, Laboratoire de Physique des Plasmas, CNRS-Ecole Polytechnique-UPMC, \\ Observatoire de Saint-Maur, 4 avenue de Neptune, 94107 Saint-Maur-des-Fossés, France \\ ${ }^{3}$ Space Research Institute, Austrian Academy of Sciences, Graz, Austria
}

Received: 22 June 2011 - Revised: 5 September 2011 - Accepted: 24 October 2011 - Published: 28 October 2011

\begin{abstract}
We study one event of reconnection onset associated to a small substorm on 27 September 2006 by using Cluster observations at inter-spacecraft separation of about $10000 \mathrm{~km}$. We focus on the acceleration of suprathermal electrons during different stages of reconnection. We show that several distinct stages of acceleration occur: (1) moderate acceleration during reconnection of pre-existing plasma sheet flux tubes, (2) stronger acceleration during reconnection of lobe flux tubes, (3) production of the most energetic electrons within dipolarization fronts (magnetic pile-up regions). The strongest acceleration is reached at the location of $B_{\mathrm{Z}}$ maxima inside the magnetic pile-up region where the reconnection jet stops. Very strong localized dawn-dusk electric field are observed within the magnetic pile-up regions and are associated to most of the magnetic flux transport.
\end{abstract}

Keywords. Magnetospheric physics (Magnetotail) - Space plasma physics (Magnetic reconnection)

\section{Introduction}

Suprathermal tails in electron distribution functions are commonly observed in the Earth magnetotail and their presence has been associated to the magnetic reconnection process (Terasawa and Nishida, 1976; Hoshino et al., 2001). There are different mechanisms accounting for the energization of such electrons. Electrons are thought to be first accelerated by the reconnection electric field at the X-line (Øieroset et al., 2002; Pritchett, 2006; Retinò et al., 2008) and then further accelerated around the Xline within magnetic flux pile-up regions (Hoshino et al.,

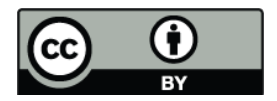

Correspondence to: A. Vaivads (andris@irfu.se)
2001; Imada et al., 2007) and/or magnetic islands (Pritchett, 2008; Retinò et al., 2008; Oka et al., 2010). At larger spatial scales, betatron acceleration is efficient enough to account for the observed fluxes of energetic electrons (Åsnes et al., 2008) as confirmed by recent observations and simulations (Khotyaintsev et al., 2011; Ashour-Abdalla et al., 2011), although other studies indicate that the acceleration mechanisms can be more complex (Asano et al., 2010; Retinò et al., 2011).

The most energetic suprathermal tails are very often associated to the magnetic pile-up regions forming ahead of fast earthward reconnection jets (Khotyaintsev et al., 2011; Fu et al., 2011). Pile-up region forms close to the reconnection site and can propagate relatively undisturbed over large distance, $-20 R_{\mathrm{E}}$ to $-11 R_{\mathrm{E}}$ (Nakamura et al., 2009; Runov et al., 2009). The front edge of magnetic flux pileup regions is often in literature referred to as dipolarization front. Such regions can have sub-ion-scale structure associated to strong DC electric fields and electromagnetic fluctuations however their role in suprathermal electron acceleration is not yet clear (Sergeev et al., 2009; Retinò et al., 2011).

Here we present one case of Cluster observations where energization of electron suprathermal tails to different energies can be explained by different stages of the reconnection in the magnetotail.

\section{Observations}

\subsection{Event overview}

We present Cluster observations during the time period 27 September 2006 17:10-17:26 UT. Cluster is located in the tail with inter-spacecraft separation of about $10000 \mathrm{~km}$, see Fig. 1. C4 is the closest to the Earth. All the satellites are located slightly towards the evening side from the midnight

Published by Copernicus Publications on behalf of the European Geosciences Union. 


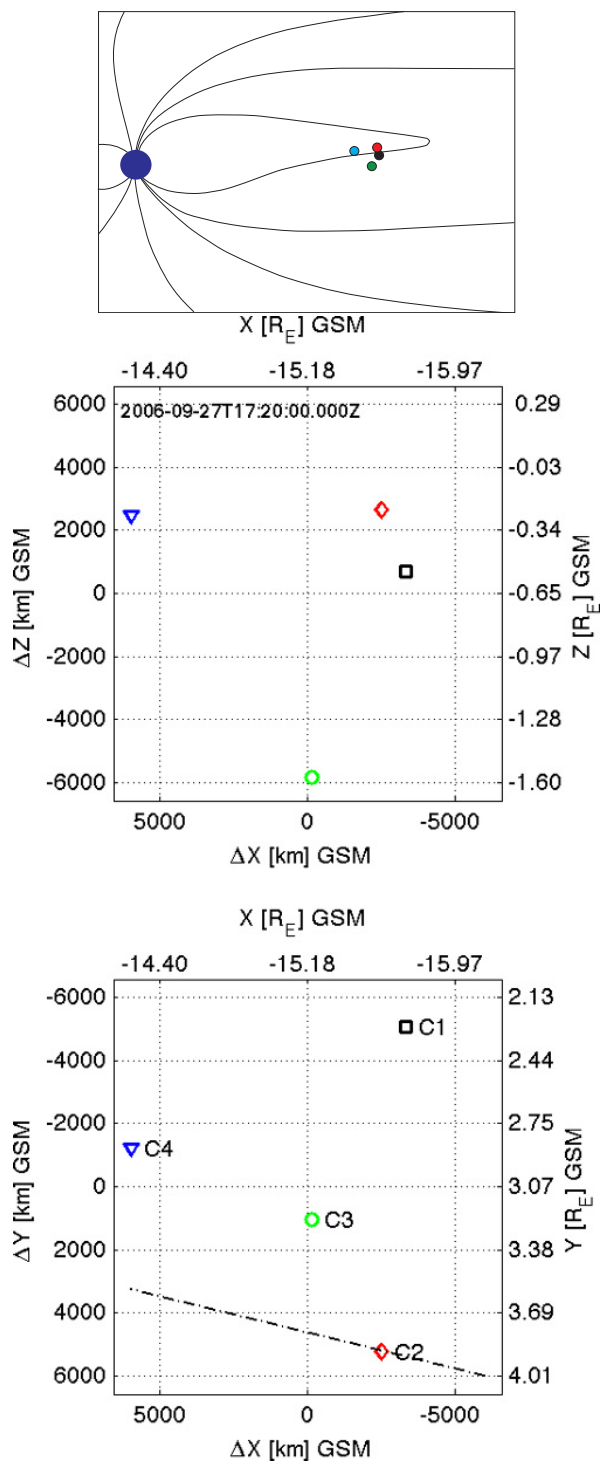

Fig. 1. Spacecraft location. Top panel shows the sketch of spacecraft location within the magnetosphere. The bottom panel show the spacecraft configuration in GSM reference frame. The dotted line shows the projection of the magnetic field observed by $\mathrm{C} 2$ in XY-plane.

meridional plane, with $\mathrm{C} 2$ at $Y=3.9 R_{\mathrm{E}}$ being the furthest from midnight. During the event all spacecraft were located south of the current sheet.

We use measurements of magnetic field (FGM instrument), electric field (EFW), ions (CIS) and electrons (PEACE, RAPID) (Escoubet et al., 1997).

Figure 2 is the overview of the event as observed by $\mathrm{C} 4$, that is the spacecraft measuring the largest suprathermal electron fluxes. In the plot we have identified several characteristic time instants labelled " $\mathrm{A}$ " to " $\mathrm{F}$ " and in the text we refer to them only by their label (e.g. "after ' $A$ "" will be used instead of "after the time "A").
Figure 2a shows the magnetic field. Initially ("A") $B_{\mathrm{X}}$ is the dominant component indicating an elongated magnetotail. Around "B" the magnetic field amplitude starts to decrease and it reaches a local minimum between " $C$ " and " $D$ ", when $B_{\mathrm{Z}}$ starts to increase while $B_{\mathrm{x}}$ is approximately constant. Between "D" and " $\mathrm{E}$ " $B_{\mathrm{Z}}$ is the dominant component and the topology of tail has a more dipolar topology. Figure $2 \mathrm{~b}$ shows the proton velocity. Note that the data are noisy due to low count statistics. In the beginning of the event "A-B" no significant flows are observed and ion density is low, Fig. 2c. After "B" fast earthward flows up to $800 \mathrm{~km} \mathrm{~s}^{-1}$ are observed. Afterwards the flows decrease, but again large earthward flows occur in the interval "D"-"E". The flows become tailward in the interval "E"-"F". Fast earthward/tailward flows can be seen also during the later part of the event. Figure $2 \mathrm{~d}$ shows measurements of the suprathermal electron fluxes. The fluxes in the beginning of the interval are close to the noise level. They start to gradually increase around "B" and reach the largest values around "E". After this time interval the fluxes decrease, although one can see a few sharp variations also later during the event. Figure 2e shows the spectrogram of the differential energy flux of electrons in the thermal range $(<30 \mathrm{keV})$. In the beginning of the event low fluxes of electrons are seen and thus the satellite is within the lobe or at boundary between the lobe and the plasma sheet boundary layer. Typical electron energies are low, below $1 \mathrm{keV}$. Starting with "C" more energetic electrons (a few $\mathrm{keV}$ ) are observed and after " $\mathrm{D}$ " energies go up to $10 \mathrm{keV}$. Such energetic electrons are observed during all later part of the event.

\subsection{Ground observations}

Figure 3 shows the relation between observations on the ground and in space. Figure 3a shows the AE/AU/AL/AO indices. The onset of the substorm occurs about $1 \mathrm{~min}$ after "E", around 17:24 UT and the substorm becomes fully developed around 17:26 UT. Figure 3b shows the measurements from the equatorial station: Pi2 pulsations can be clearly seen with onset just before " $\mathrm{C}$ ". The onset of Pi2 pulsations is almost 5 min before the substorm onset. Figure $3 \mathrm{c}$ shows $B_{\mathrm{Z}}$ component from $\mathrm{C} 2$ and $\mathrm{C} 4$. The onset of $\mathrm{Pi} 2$ pulsations is about 2 min after the observations of the first increases in $B_{\mathrm{Z}}$ by $\mathrm{C} 2$. The discussion part gives the possible explanation of the observed time delays between space and ground observations.

\subsection{Multi-spacecraft observations}

Figure 4 shows comparative observations among all Cluster spacecraft. Figure 4 a shows $B_{\mathrm{Z}}$ component. All four spacecraft observe a magnetic pile-up region in the interval "D-E", where $B_{\mathrm{Z}}$ goes from a few nT to average values above $5 \mathrm{nT}$. $\mathrm{C} 4$, which is the closest to the Earth, observes the highest values of $B_{\mathrm{z}} \sim 15 \mathrm{nT}$ at " $\mathrm{E}$ ". Figure $4 \mathrm{~b}$ shows $B_{\mathrm{x}}$ component 


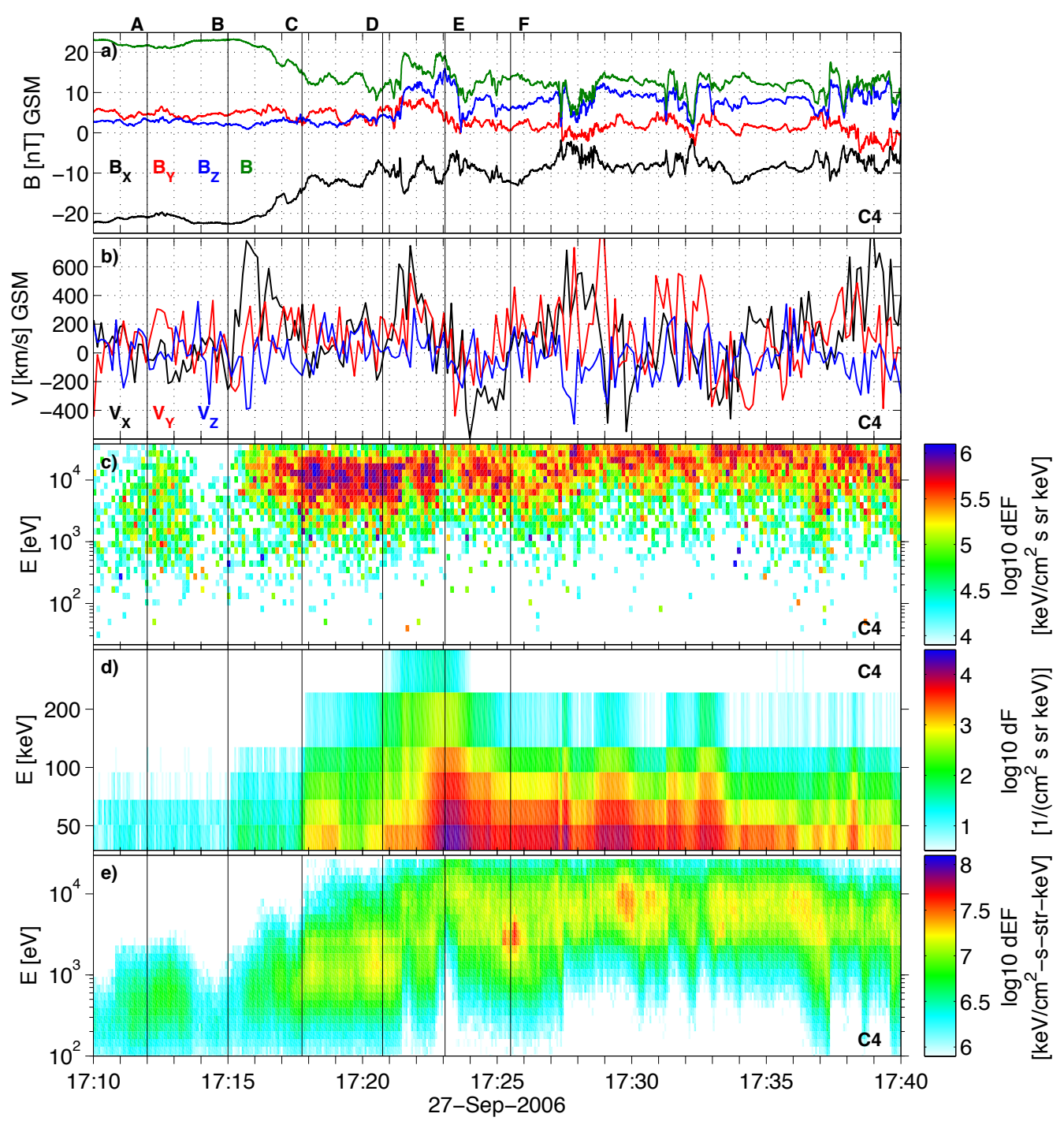

Fig. 2. Overview observations by C4. (a) magnetic field, (b) $\mathrm{H}^{+}$velocity, (c) energy spectrogram of $\mathrm{H}^{+}$ions, (d) suprathermal electron energy spectrogram (RAPID instrument), (e) electron spectrogram (PEACE instrument).

that is a good indicator of how deep in the current sheet satellites are. $\mathrm{C} 3$, according to Fig. 1, is the furthest from the current sheet and consistently it observes the most negative $B_{\mathrm{X}}$ values throughout the event.

One can see that in the interval "A-B" and around "F" all satellites observe similar magnetic fields (with small variations) while in the time interval "C-E" large differences among the spacecraft are found. This indicates that in the interval "A-B" and later around "F", there were only large scale currents flowing (on the scale of spacecraft separation $\sim 10000 \mathrm{~km})$ and at the spacecraft locations the magnetosphere changed from stretched to more dipolar magnetic field topology. On the other hand, in the interval "C-E", the situation is more dynamic and small-scale current structures are present.
Figure $4 \mathrm{c}$ shows the differential energy flux of suprathermal electrons (energy range 68-95 keV) from all spacecraft. It can bee seen that the highest fluxes are observed by $\mathrm{C} 4$, the spacecraft closest to the Earth, and that the highest flux is reached around " $E$ " when also $B_{\mathrm{Z}}$ has the highest amplitudes. This is also the time when the plasma flow, see Fig. $2 b$ is turning from being earthward to being tailward. All satellite show the same trend that the highest fluxes correlate with the time interval of highest $B_{\mathrm{Z}}$ values. It is also seen that the initial increase in suprathermal electron fluxes occurs stepwise. The first stepwise increase is observed almost simultaneously by $\mathrm{C} 2$ and $\mathrm{C} 4$ at "B" and the second stepwise increase is seen by $\mathrm{C} 2$ and $\mathrm{C} 4$ a few minutes later at "C". In addition, $\mathrm{C} 2$ observes additional stepwise increase at " $\mathrm{D}$ ", while flux increase seen by $\mathrm{C} 4$ during that time is more smooth. Also $\mathrm{C} 1$ and $\mathrm{C} 3$ observe stepwise increases in electron fluxes but 

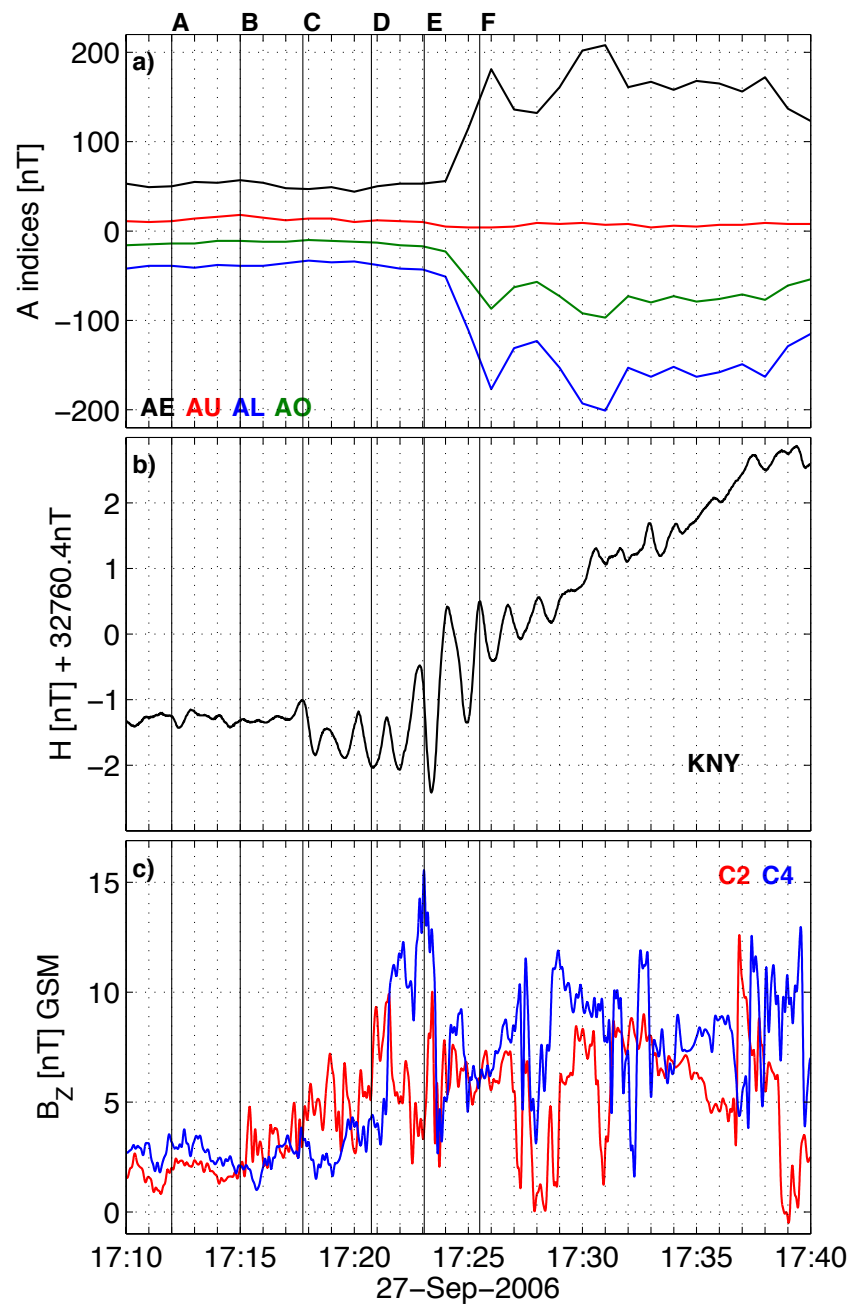

Fig. 3. Correlation between space and ground observations. (a) AE/AU/AL/AO indices, (b) Kanoya stations ground magnetometer data, (c) $B_{\mathrm{Z}}$ component from $\mathrm{C} 2$ and $\mathrm{C} 4$.

those are not correlated to the flux increases observed by $\mathrm{C} 2$ and $\mathrm{C} 4$, except for the one observed by $\mathrm{C} 1$ around " $\mathrm{D}$ ".

Figure $4 \mathrm{~d}$ shows the time integral of dawn dusk electric field as observed by satellites $\mathrm{C} 1, \mathrm{C} 2$ and $\mathrm{C} 4$. The data from $\mathrm{C} 3$ are not shown because it spends most of the time far out in the lobes where the electric field measurements by the EFW instrument suffers from wake effects. To make easier the interpretation of the dawn-dusk electric field time integral we present it in the units of magnetic flux transport. The electric field in general has the same unit as magnetic flux transport. The dawn dusk electric field corresponds to the magnetic flux transport in the $\mathrm{X}-\mathrm{Z}$ plane. When magnetic field is predominantly in Z-direction it corresponds to transport in earthward or anti-earthward direction and when magnetic field is predominantly in X-direction it corresponds to transport in north or south direction. The time integral of electric field can be expressed as the distance travelled by a

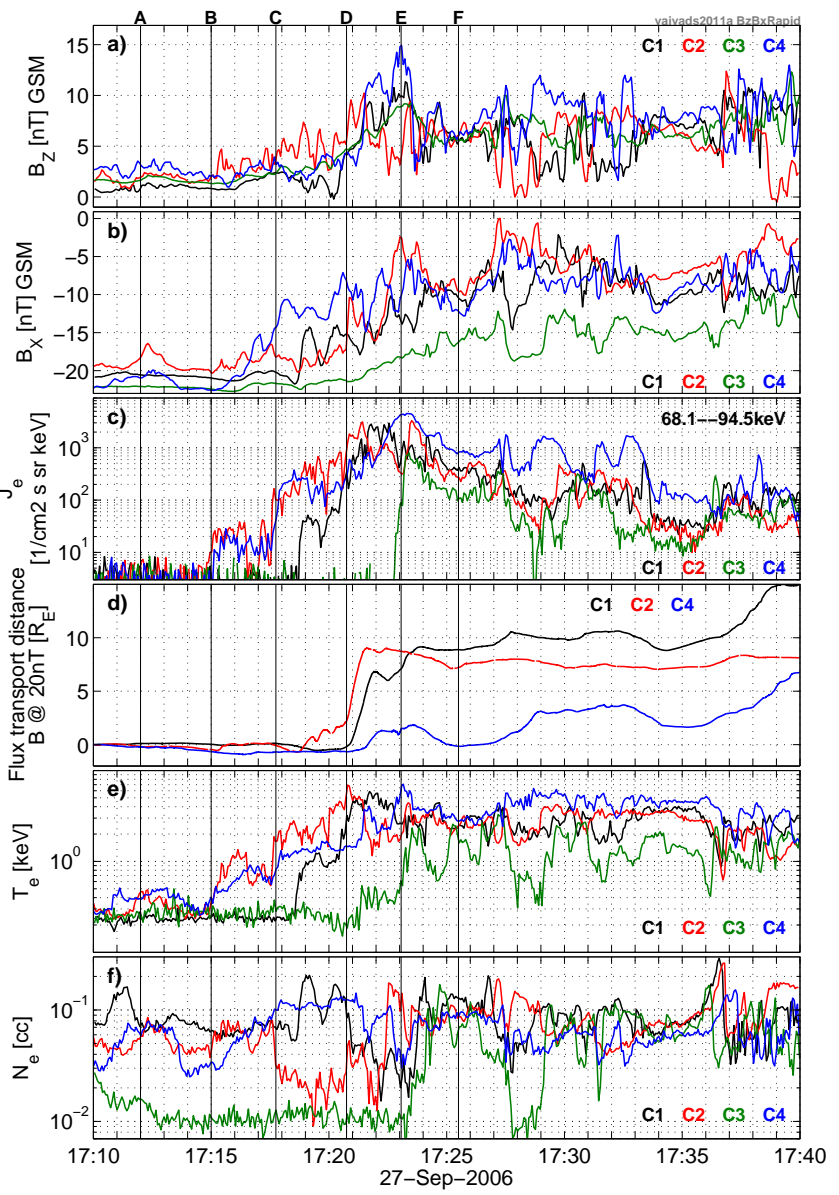

Fig. 4. Overview observations by all four Cluster spacecraft. (a) $B_{\mathrm{Z}}$, (b) $B_{\mathrm{X}}$, (c) suprathermal energy flux at one energy channel of RAPID instrument, (d) integral of $E_{\mathrm{y}}$ expressed in units of magnetic flux transport (distance that magnetic flux tubes would be transported if magnetic field magnitude was $20 \mathrm{nT}$, a typical value in the lobe), (e) electron temperature, (f) electron density.

magnetic flux tubes. In our case, the magnetic flux transport we measure as how large distance we would need to transport magnetic flux tubes having magnetic field amplitude of $20 \mathrm{nT}$. In our event, this is a typical strength of magnetic field in the lobe around "A". Figure $4 \mathrm{~d}$ shows that on $\mathrm{C} 1$ and $\mathrm{C} 2$ there is large jump (around $10 R_{\mathrm{E}}$ ) just after "D" when magnetic flux pileup region hits $\mathrm{C} 1$ and $\mathrm{C} 2$. Integrating corresponding dawn-dusk electric field between $\mathrm{C} 1$ and $\mathrm{C} 2$ at the location of the jump would give potential drop of the order several hundred $\mathrm{kV}$. The positive jump corresponds to the magnetic flux transport across the spacecraft in the earthward direction. However, $\mathrm{C} 4$ does not observe such jump. $\mathrm{C} 4 \mathrm{ob}-$ serves a few $R_{\mathrm{E}}$ increase after "D", corresponding to earthward transport of magnetic flux in the beginning of magnetic flux pileup region, and a few $R_{\mathrm{E}}$ decrease after "E", corresponding to tailward transport of magnetic flux at the end of magnetic flux pileup region. The later increases at $\mathrm{C} 4$ after 
"F" seem to be associated with the next pileup regions passing C4. In summary, during the time interval from " $\mathrm{A}$ " to "F", there is large amount of magnetic flux passing $\mathrm{C} 1$ and $\mathrm{C} 2$ in earthward direction while the total amount passing $\mathrm{C} 4$ is close to zero.

Finally, Fig. 4e, f shows the temperature and density of electrons as observed by all spacecraft. In the beginning of the interval, temperature is around $200 \mathrm{eV}$. Spacecraft C3 is furthest out in the lobe and observes the lowest densities of $\sim 0.01 \mathrm{~cm}^{-3}$. After " $\mathrm{B}$ " electron temperature on $\mathrm{C} 2$ and $\mathrm{C} 4$ increases to $400-900 \mathrm{eV}$. After " $\mathrm{C}$ " there is an additional increase in electron temperature, up to $\sim 1 \mathrm{keV}$ on $\mathrm{C} 4$ and $\sim 2 \mathrm{keV}$ on $\mathrm{C} 2$. There is additional increase of temperature reaching peak values up to $4-5 \mathrm{keV}$ around " $\mathrm{D}$ " on $\mathrm{C} 2$ and "E" on C4. During this time, "D"-"E", temperature is very varying. Later, at "F" temperature is back to $\sim 2 \mathrm{keV}$ and density is $\sim 0.1 \mathrm{~cm}^{-3}$, typical values for plasma sheet.

\subsection{Poynting flux}

Figure 5 shows the Poynting flux associated with the event. Figure 5a,b shows magnetic and electric field measurements by $\mathrm{C} 2$ and Fig. 5c shows the Poynting flux. We look at the Poynting flux associated to disturbances in electric and magnetic fields and constructed in the following way: the data are low-pass filtered below wave period $1 \mathrm{~s}$ and then background field is obtained low-pass filtering the data below wave period $2 \mathrm{~min}$. The disturbance fields are obtained by subtracting background fields from the full measurements. In Fig. 5 all the quantities are shown in ISR2 (Inverted Spacecraft Reference) reference frame which has $\mathrm{X}$ - and Y-components in the satellite spin plane. ISR2 reference frame is very close to GSE reference frame (difference is less than $3.5 \mathrm{deg}$ ). The reason for using ISR2 reference frame is that EFW instrument measures only 2 components of electric field ( $E_{\mathrm{X}}$ and $E_{\mathrm{Y}}$ in ISR2) and staying in ISR2 reference frame we have better control over all possible error sources (Khotyaintsev et al., 2010). In this event the third component of electric field $\left(E_{\mathrm{Z}}\right)$ cannot be reconstructed from the assumption $\boldsymbol{E} \times \boldsymbol{B}=0$ because mangetic field was close to the spin plane most of the event. Thus, when estimating the components of the Poynting flux, $S_{Z}$ is fully estimated while the contribution to $S_{\mathrm{X}}$ and $S_{\mathrm{Y}}$ from $E_{\mathrm{Z}}$ cannot be estimated. Thus if we assume that $E_{\mathrm{Z}}$ contributes roughly as much to the Poynting flux as other components, then most probably we are underestimating the total $S_{\mathrm{X}}$ and $S_{\mathrm{Y}}$ by a factor of 2 .

Figure 5c shows that there is almost continuous Poynting flux in the positive X-direction (towards the Earth) that starts just after "B" and there is also Poynting flux in the negative Z-direction (away from the current sheet). To see the cumulative effect of the Poynting flux, in Fig. 5d we show the time integral of the Poynting flux. Finally, in Fig. 5e we show the X component of the integrated Poynting flux from all 4 spacecraft. A clear eartwhward Poynting flux can be seen on three of the spacecraft, the ones which are closer to the cur-

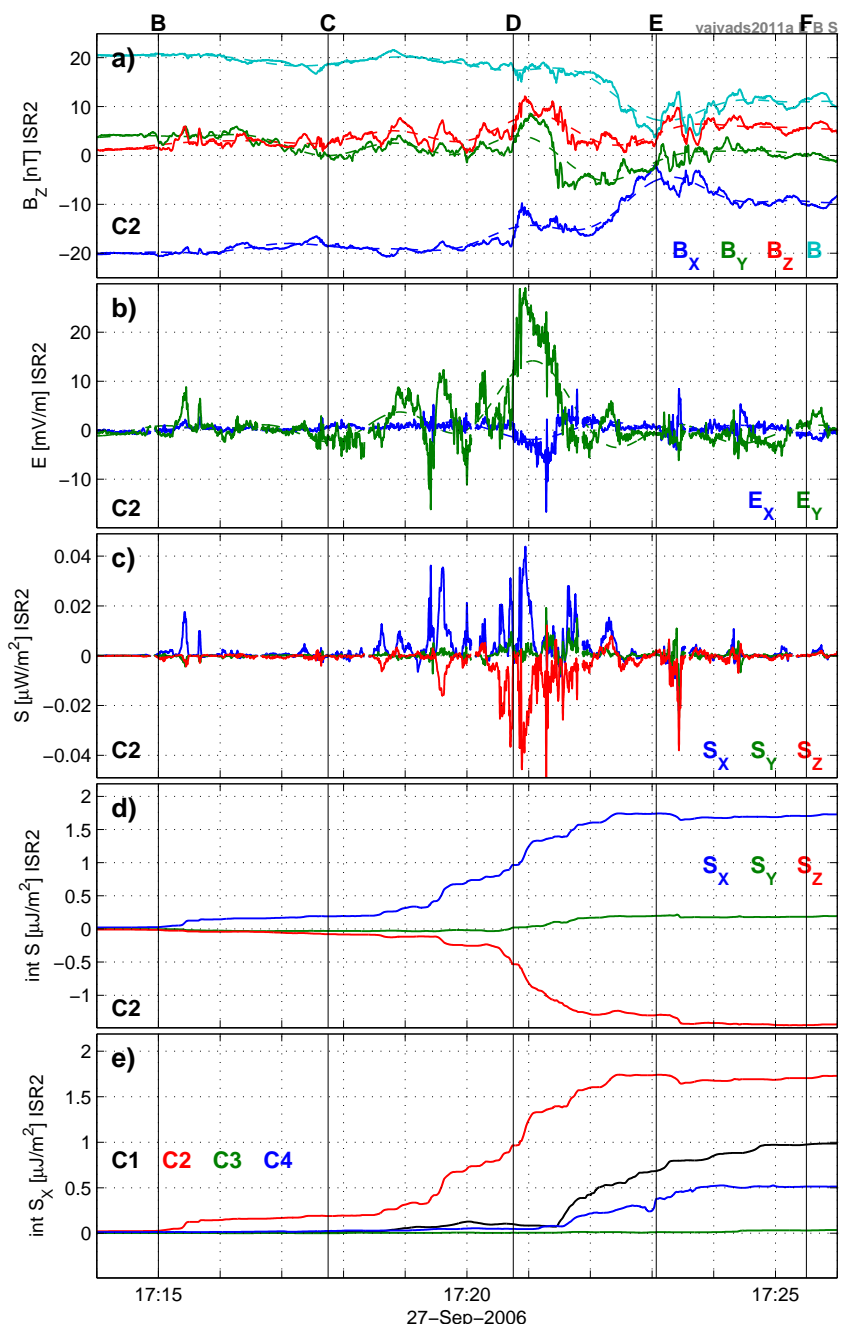

Fig. 5. Poynting flux observations. (a) total magnetic field (solid) and background magnetic field (dashed), (b) total electric field and background electric field(dashed), (c) Poynting flux, (d) integrated Poynting flux, (e) X component of integrated Poynting flux from all spacecraft.

rent sheet. C3 is mainly out in the lobe and does not observe significant Poynting flux. C2 sees the largest Poynting flux of all spacecraft and it is also the spacecraft that first observes the increase of the Poynting flux at " $\mathrm{B}$ ".

\section{Discussion}

The observations in Fig. 4d show that during the event there are several consequent suprathermal electron energization steps, indicating that there are several and most probably different energization mechanisms at work. In Fig. 6 we show a cartoon of the event to explain our interpretation of suprathermal electron acceleration at each different stage of reconnection. 

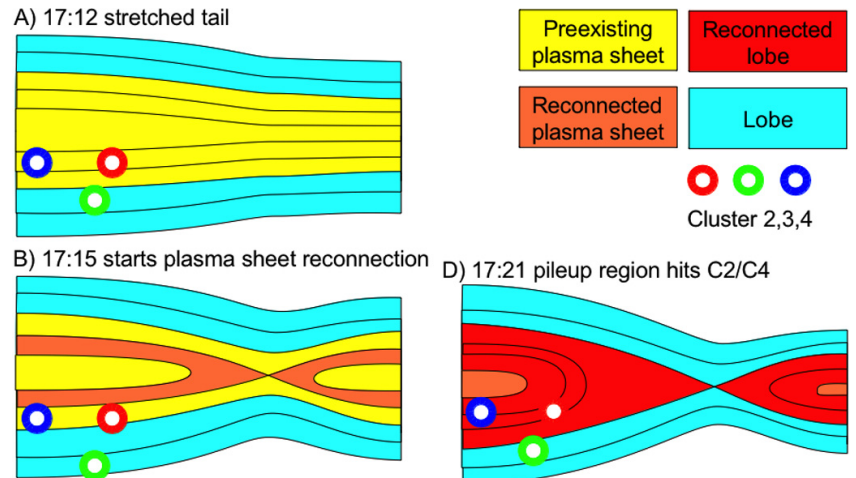

C) $17: 18$ starts lobe reconnection
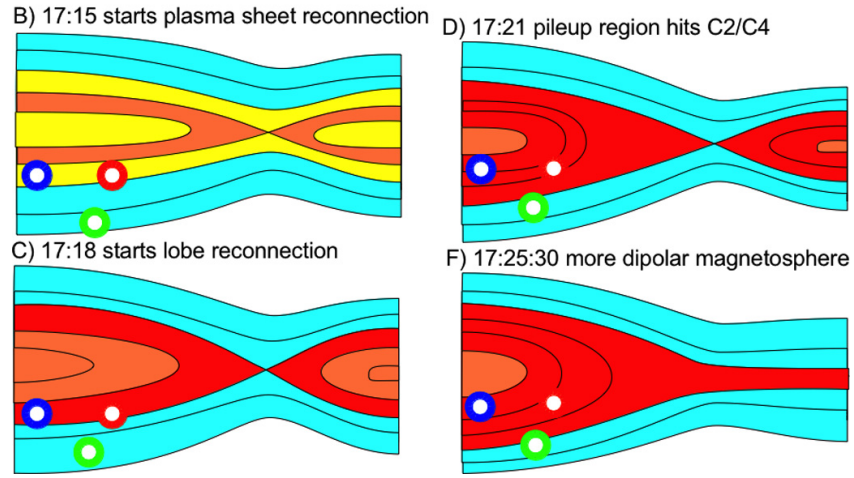

F) 17:25:30 more dipolar magnetosphere

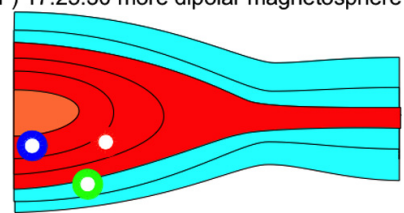

Fig. 6. Sketch of the temporal evolution of reconnection process during the event.

The color in Fig. 6 represents the temperature of the plasma, where blue is the coldest and red is the hottest. From "A" to "F", at the location of spacecraft, the magnetotail configuration changes from stretched into more dipolar. Initially satellites observe relatively cold electrons (less than $1 \mathrm{keV}$ ), most probably indicative of pre-existing cold plasma sheet (colored yellow). Reconnection starts at "B" when C2 and $\mathrm{C} 4$ observe the first energetic electrons (Fig. 4c) and $\mathrm{C} 2 \mathrm{ob}-$ serves the first earthward Poynting flux (Fig. 5c). At this stage, reconnection occurs between plasma sheet flux tubes and the observed energetic electrons most probably come directly from the reconnection site where they gained most of their energy. This is also consistent with $\mathrm{C} 2$ and $\mathrm{C} 4$ observing similar flux levels after "B". C3 does not observe increase because it is located out in the lobes while $\mathrm{C} 1$ is likely is located too far on the dawn side and thus is not observing the magnetic flux tubes mapping into the reconnection region. Note that $\mathrm{C} 2$ and $\mathrm{C} 4$ are separated in the Y-direction by more than $1 R_{\mathrm{E}}$, however if we take into account the observed orientation of magnetic field (shown in Fig. 1) the separation between their magnetic flux tubes is smaller.

After all plasma sheet flux tubes have reconnected (at the spacecraft location), lobe flux tubes start to reconnect and the reconnection rate increases. This happens because the reconnection rate scales with the Alfvén speed in the inflow region and inflowing lobe plasma has much lower density than plasma sheet plasma (thus much higher Alfvén speed). Typical numbers for our case would give Alfvén speed of $\sim 4400 \mathrm{~km} \mathrm{~s}^{-1}$ in lobes $\left(B=20 \mathrm{nT}, n=0.01 \mathrm{~cm}^{-3}\right)$ and $\sim 1000 \mathrm{~km} \mathrm{~s}^{-1}$ in plasma sheet $\left(B=15 \mathrm{nT}, n=0.1 \mathrm{~cm}^{-3}\right)$. The increase in the reconnection rate corresponds to large electric fields around the reconnection site and can be the cause of the subsequent jump in the suprathermal electron fluxes at "C". This jump again is observed only by $\mathrm{C} 2$ and
$\mathrm{C} 4$, indicating that the magnetic flux tubes observed by those spacecraft map closer to the reconnection site. The simultaneous suprathermal electron flux increase observed by $\mathrm{C} 2$ and $\mathrm{C} 4$ at "C" indicates that this increase can not be a spatial effect of spacecraft crossing some boundary; $\mathrm{C} 2$ and $\mathrm{C} 4$ are located far apart and density observations in Fig. 4 even indicate that $\mathrm{C} 2$ and $\mathrm{C} 4$ move in opposite direction with respect to plasma sheet. $\mathrm{C} 2$ and $\mathrm{C} 4$ also observes an increase in the electron temperature at " $\mathrm{C}$ ", that is consistent with the above interpretation since higher electron temperature is expected during lobe reconnection due to higher Alfvén speed in lobes. It has been shown in numerical simulations and confirmed by observations that electron acceleration near X-line scales with the inflow Alfvén speed (Hoshino et al., 2001).

Finally, the last jump in the suprathermal electron fluxes is observed around "D" for C2 and slightly after " $D$ " for $C 4$. This jump corresponds to the largest fluxes observed by both spacecraft, reaching the peak by $\mathrm{C} 4$ at "E". This last step of flux increase is consistent with acceleration in the magnetic flux pile-up region, that propagates earthward from the reconnection site reaching $\mathrm{C} 2$ and slightly later $\mathrm{C} 4$. While at $\mathrm{C} 2$ the magnetic flux pileup region is passing very fast (speeds larger than $1000 \mathrm{~km} \mathrm{~s}^{-1}$ estimated from $\boldsymbol{E} \times \boldsymbol{B}$, not shown, at $\mathrm{C} 4$ the speeds is much slower and after " $\mathrm{E}$ " the flow even reverses its direction. The flow reversal at $\mathrm{C} 4$ observed around "E" can be clearly seen in Fig. $4 d$ as well as in Fig. 2b. Thus the peak in the magnetic flux at $\mathrm{C} 4$ is observed when there is a reversal of magnetic flux transport from earthward to tailward and this also coincides with the time interval of highest fluxes in suprathermal electrons. This means that the pile-up region decelerates in between $\mathrm{C} 2$ and $\mathrm{C} 4$ and stops at $\mathrm{C} 4$, indicating that Cluster is in the flow braking region (Retinò et al., 2011). The timing of the pile-up region between $\mathrm{C} 2$ and $\mathrm{C} 4$ is not possible due to very different profiles of the pile-up region, indicating that the such region is likely not planar over the separation $\mathrm{C} 2-\mathrm{C} 4$ in Y-direction and/or that the region is not stationary in time over the propagation.

The proposed evolution of reconnection in the magnetotail is also supported by ground observations. Figure $3 \mathrm{a}$ with the AE/AU/AL/AO indices shows that the onset of the substorm is about $1 \mathrm{~min}$ after "E", around 17:24 UT, and the substorm is fully developed around 17:26 UT. The Alfvénic propagation time from the magnetosphere at position of $\mathrm{C} 4$ down to the ionosphere is of the order 2 min and thus it is consistent with the substorm onset being related to the full braking of the lobe reconnection jet close to the Earth. The onset time of $\mathrm{Pi} 2$ pulsations, shown in Fig. 3b, is just before " $\mathrm{C}$ ". The onset time of $\mathrm{Pi} 2$ pulsations is about $2 \mathrm{~min}$ after the observations of the first $B_{\mathrm{Z}}$ increase at $\mathrm{C} 2$, shown in Fig. 3c. The propagation speed of $B_{\mathrm{Z}}$ disturbances, as estimated earlier, is more than $1000 \mathrm{~km} \mathrm{~s}^{-1}\left(1 R_{\mathrm{E}}\right.$ in $\left.\sim 5 \mathrm{~s}\right)$ and thus it would take more than $1 \mathrm{~min}$ for the $B_{\mathrm{Z}}$ disturbances to propagate down to the equatorial ionosphere (assuming the same Alfvén speed). Thus the onset of Pi2 is most probably associated with the onset of the plasma sheet reconnection 
in the tail. At the same time one can notice that the largest amplitude of $\mathrm{Pi} 2$ oscillations agree with the onset of the substorm and thus are correlated in time with the reconnection jet braking in the near Earth region. Similar relation between $\mathrm{Pi} 2$ oscillations and the onset of the substorm has been observed earlier, where it is shown that magnetic waves on the time scale 1-40 s occur a few minutes before the onset of the the substorm (Rae et al., 2009). Although that study gives a different interpretation of the data ("inside-out" scenario of the substorm), it can also be consistent with the model presented here where initial reconnection in pre-existing plasma sheet is followed by the lobe reconnection leading to strong auroral arcs. Association between $\mathrm{Pi} 2$ oscillations and $B_{\mathrm{Z}}$ fluctuations related to impulsive reconnection in the tail observed by Cluster satellites has been also shown by Keiling et al. (2006).

Finally, we address the question of suprathermal electron energization around the time when their highest fluxes are observed. In Fig. 7 we zoom into this region. Figure $7 \mathrm{a}$ shows $B_{\mathrm{Z}}$ component, the highest value is observed by $\mathrm{C} 4$ at "E". Next panels, Fig. 7b-e show the pitch angle spectrograms for $\mathrm{C} 1-\mathrm{C} 4$. First, it can be seen that the highest suprathermal electron fluxes are observed by $\mathrm{C} 4$, as previously mentioned. Furthermore, the $\mathrm{C} 4$ data show that the acceleration is more in perpendicular direction than parallel/antiparallel directions, suggesting betatron acceleration (Asano et al., 2010). On the other hand C2 observes electron fluxes in perpendicular direction that are sometimes larger and sometimes smaller than those in the parallel/antiparallel directions. One possible speculation is that Fermi acceleration occurs together with betatron. The conditions that favor one or other mechanism has been shown in a recent study by Fu et al. (2011).

We focus now in more detail on the observations of the peak suprathermal electron fluxes by C4. Detailed comparison shows that the peak fluxes are not observed exactly at "E" but slightly later. In particular, the peek seems to be associated with a local $B_{\mathrm{Z}}$ dip about $15 \mathrm{~s}$ after "E". Recent study by Khotyaintsev et al. (2011) suggests that whistler emissions can be a good indicator of locations where active betatron acceleration is ongoing. Therefore, in the next panel, Fig. 7f, we plot magnetic field spectrogram. The black line shows the electron gyrofrequency. Whistler waves are expected to be observed in the frequency range from about $50 \mathrm{~Hz}$ to the electron gyrofrequency. Indeed, localized whistler emissions around $100 \mathrm{~Hz}$ can be seen being correlated with the two local $B_{\mathrm{Z}}$ dips after "E". This suggest that suprathermal electron acceleration within the magnetic pile-up region can be dependent on the internal structure and dynamics of the magnetic pile-up region on smaller scales, as also found for other events (Retinò et al., 2011). This stresses the importance to carry out in future detailed studies of the electron acceleration mechanisms on suchsmall scales, similarly to what has been done in thin current sheets in magnetotail reconnection regions (Retinò et al., 2008).

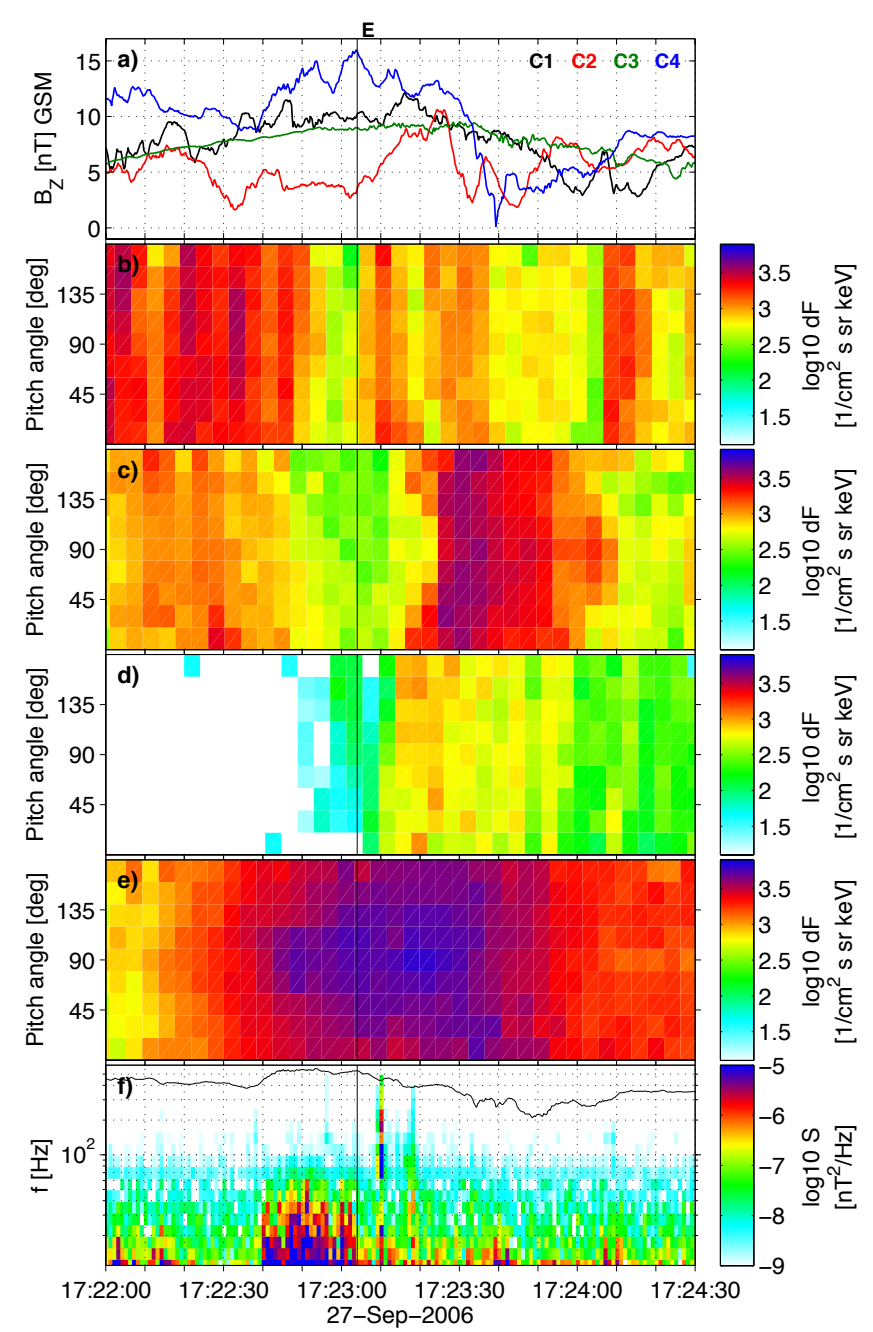

Fig. 7. Observations around the time of highest suprathermal electron fluxes. (a) $B_{\mathrm{Z}}$, (b)-(e) pitch angle spectrogram of electron flux in one energy channel $(42-64 \mathrm{keV})$ of RAPID instrument, (f) magnetic field frequency spectrogram, line marks electron gyrofrequency.

\section{Summary and conclusions}

We have presented observations with Cluster multispacecraft that are located at large separation $(10000 \mathrm{~km})$ in the magnetotail around the time of reconnection/substorm onset.

Our observations are consistent with different reconnection steps, each associated to a different suprathermal electron acceleration mechanisms: (1) reconnection of preexisting plasma sheet with absence of reconnection jet braking region, (2) onset of lobe reconnection, (3) formation of reconnection jet braking region associated with strong magnetic flux pile-up. Stage (1) is associated with Earthward Poynting flux on the field lines connected to the reconnection region. Following the onset of lobe reconnection, strong 
dawn-dusk $E_{\mathrm{y}}$ fields are observed associated to pile-up regions, clearly showing that most of the magnetic flux transport is associated with the earthward propagation of such regions. Potentials of several hundred $\mathrm{kV}$ over the the spacecraft separation of about $10000 \mathrm{~km}$ are associated to the jet braking front.

Ground observations of auroral indices and Pi2 oscillations are consistent with the observed several steps of reconnection: (1) Pi2 oscillation onset is related to plasma-sheet reconnection onset, with the highest amplitudes associated to the formation of the jet braking region; (2) substorm onset observed in the auroral indices is related to the onset of lobe reconnection; when the jet braking region forms, substorm becomes fully developed.

Our observations based on the RAPID instrument (electron energies above $40 \mathrm{keV}$ ) are consistent with the interpretation that in the suprathermal electron energization one can clearly distinguish several stages: (1) relatively small increase when reconnection of the pre-existing plasma sheet starts. (2) Significant increase when the reconnection of lobe plasma starts. (3) Largest fluxes observed in magnetic pileup boundary with the maximum flux throughout the event being in the region with strongest magnitude of the magnetic field in the magnetic pile-up region, coinciding also with the region where reconnection jet is fully stopped and bounces back.

Acknowledgements. Guest Editor A. Masson thanks M. AshourAbdalla and another anonymous referee for their help in evaluating this paper.

\section{References}

Åsnes, A., Friedel, R. W. H., Lavraud, B., Reeves, G. D., Taylor, M. G. G. T., and Daly, P.: Statistical properties of tail plasma sheet electrons above $40 \mathrm{keV}$, J. Geophys. Res., 113, A03202, doi:10.1029/2007JA012502, 2008.

Asano, Y., Shinohara, I., Retinò, A., Daly, P. W., Kronberg, E. A., Takada, T., Nakamura, R., Khotyaintsev, Y. V., Vaivads, A., Nagai, T., Baumjohann, W., Fazakerley, A. N., Owen, C. J., Miyashita, Y., Lucek, E. A., and Rème, H.: Electron acceleration signatures in the magnetotail associated with substorms, J. Geophys. Res., 115, 5215, doi:10.1029/2009JA014587, 2010.

Ashour-Abdalla, M., El-Alaoui, M., Goldstein, M. L., Zhou, M., Schriver, D., Richard, R., Walker, R., Kivelson, M. G., and Hwang, K.-J.: Observations and simulations of non-local acceleration of electrons in magnetotail magnetic reconnection events, Nature Physics, 7, 360-365, doi:10.1038/nphys1903, 2011.

Escoubet, C. P., Schmidt, R., and Goldstein, M. L.: Cluster Science and Mission Overview, Space Sci. Rev., 79, 11-32, doi:10.1023/A:1004923124586, 1997.

Fu, H. S., Khotyaintsev, Y. V., André, M., and Vaivads, A.: Fermi and betatron acceleration of suprathermal electrons behind dipolarization fronts, Geophys. Res. Lett., 381, L16104, doi:10.1029/2011GL048528, 2011.
Hoshino, M., Mukai, T., Terasawa, T., and Shinohara, I.: Suprathermal electron acceleration in magnetic reconnection, J. Geophys. Res., 106, 25979-25998, doi:10.1029/2001JA900052, 2001.

Imada, S., Nakamura, R., Daly, P. W., Hoshino, M., Baumjohann, W., Mühlbachler, S., Balogh, A., and Rème, H.: Energetic electron acceleration in the downstream reconnection outflow region, J. Geophys. Res., 112, A03202, doi:10.1029/2006JA011847, 2007.

Keiling, A., Fujimoto, M., Hasegawa, H., Honary, F., Sergeev, V., Semenov, V. S., Frey, H. U., Amm, O., Rème, H., Dandouras, I., and Lucek, E.: Association of Pi2 pulsations and pulsed reconnection: ground and Cluster observations in the tail lobe at $16 R_{E}$, Ann. Geophys., 24, 3433-3449, doi:10.5194/angeo-243433-2006, 2006.

Khotyaintsev, Y., Lindqvist, P., Eriksson, A., and André, M.: The EFW Data in the CAA, in: The Cluster Active Archive, Studying the Earth's Space Plasma Environment, edited by: Laakso, H., Taylor, M., and Escoubet, C. P., pp. 97-108, Springer, doi:10.1007/978-90-481-3499-1_6, 2010.

Khotyaintsev, Y. V., Cully, C. M., Vaivads, A., André, M., and Owen, C. J.: Plasma Jet Braking: Energy Dissipation and Nonadiabatic Electrons, Phys. Rev. Lett., 106, 165001, doi:10.1103/PhysRevLett.106.165001, 2011.

Nakamura, R., Retinò, A., Baumjohann, W., Volwerk, M., Erkaev, N., Klecker, B., Lucek, E. A., Dandouras, I., André, M., and Khotyaintsev, Y.: Evolution of dipolarization in the nearEarth current sheet induced by Earthward rapid flux transport, Ann. Geophys., 27, 1743-1754, doi:10.5194/angeo-27-17432009, 2009.

Øieroset, M., Lin, R. P., Phan, T. D., Larson, D. E., and Bale, S. D.: Evidence for Electron Acceleration up to $300 \mathrm{keV}$ in the Magnetic Reconnection Diffusion Region of Earth's Magnetotail, Phys. Rev. Lett., 89, 195001, doi:10.1103/PhysRevLett.89.195001, 2002.

Oka, M., Fujimoto, M., Shinohara, I., and Phan, T. D.: "Island surfing" mechanism of electron acceleration during magnetic reconnection, J. Geophys. Res., 115, A08223, doi:10.1029/2010JA015392, 2010.

Pritchett, P. L.: Relativistic electron production during driven magnetic reconnection, Geophys. Res. Lett., 33, L13104, doi:10.1029/2005GL025267, 2006.

Pritchett, P. L.: Energetic electron acceleration during multiisland coalescence, Physics of Plasmas, 15, 102105, doi:10.1063/1.2996321, 2008.

Rae, I. J., Mann, I. R., Angelopoulos, V., Murphy, K. R., Milling, D. K., Kale, A., Frey, H. U., Rostoker, G., Russell, C. T., Watt, C. E. J., Engebretson, M. J., Moldwin, M. B., Mende, S. B., Singer, H. J., and Donovan, E. F.: Near-Earth initiation of a terrestrial substorm, J. Geophys. Res., 114, 07220, doi:10.1029/2008JA013771, 2009.

Retinò, A., Nakamura, R., Vaivads, A., Khotyaintsev, Y., Hayakawa, T., Tanaka, K., Kasahara, S., Fujimoto, M., Shinohara, I., Eastwood, J. P., André, M., Baumjohann, W., Daly, P. W., Kronberg, E. A., and Cornilleau-Wehrlin, N.: Cluster observations of energetic electrons and electromagnetic fields within a reconnecting thin current sheet in the Earth's magnetotail, J. Geophys. Res., 113, 12215, doi:10.1029/2008JA013511, 2008.

Retinò, A., Nakamura, R., Zieger, B., Vaivads, A., Keika, K., 
Asano, Y., Khotyaintsev, Y., Kasahara, S., Fujimoto, M., Baumjohann, W., Volwerk, M., Panov, E., Andre, M., Kronberg, E. A., Daly, P., Owen, C. J., Dandouras, J., Cornilleau-Wehrlin, N., and Lucek, E. A.: Energetic Electron Acceleration in the Near-Earth Flow Braking Region: Cluster Multi-Scale Observations, J. Geophys. Res., submitted, 2011.

Runov, A., Angelopoulos, V., Sitnov, M. I., Sergeev, V. A., Bonnell, J., McFadden, J. P., Larson, D., Glassmeier, K., and Auster, U.: THEMIS observations of an earthwardpropagating dipolarization front, Geophys. Res. Lett., 36, 14106, doi:10.1029/2009GL038980, 2009.
Sergeev, V., Angelopoulos, V., Apatenkov, S., Bonnell, J., Ergun, R., Nakamura, R., McFadden, J., Larson, D., and Runov, A.: Kinetic structure of the sharp injection/dipolarization front in the flow-braking region, Geophys. Res. Lett., 36, 21105 , doi:10.1029/2009GL040658, 2009.

Terasawa, T. and Nishida, A.: Simultaneous observations of relativistic electron bursts and neutral-line signatures in the magnetotail, Planet. Space Sci., 24, 855-866, doi:10.1016/00320633(76)90076-3, 1976. 